\title{
Responsabilidad social corporativa en las entidades financieras españolas
}

\author{
Rafael Becerra-Vicario ${ }^{1}$ \\ Ana León Gómez ${ }^{2}$
}

Recibido: 29 de octubre de 2019

Aprobado: 13 de noviembre de 2019

Becerra-Vicario, R. y León Gómez, A. (2020). Responsabilidad Social Corporativa en las entidades financieras españolas. Revista Activos, 18(1), 39-75. DoI: https://https://doi.org/10.15332/25005278/6154

\section{Clasificación JEL: M14, G20}

\section{Resumen}

Esta investigación tiene por objetivo analizar las prácticas responsables desarrolladas por entidades financieras, referidas a la responsabilidad social corporativa (RCS), y determinar si el grado de intensidad en la ejecución de esas políticas responsables está relacionado con el tamaño o la solvencia de estas.

1 Profesor del Departamento de Finanzas y Contabilidad, Universidad de Málaga. Doctor en Administración de Empresas. Director de la Cátedra de Investigación Inmobiliaria. Correo electrónico: rbecerra@uma.es ORCID: https://orcid.org/0000-0002-1227-8988

2 Programa de Doctorado Interuniversitario en Turismo, Universidad de Málaga. Investigadora en la Cátedra de Investigación Inmobiliaria. Correo electrónico: ana.leon@uma.es ORCID: https://orcid.org/0000-0002-6141-9003 
Desde un enfoque positivista, a través de los principales estándares de medición de la RCs, se realizó la validación de algunas hipótesis basadas en un análisis previo de carácter empírico. Se utilizó una muestra de entidades financieras para comprender cuál es el grado de compromiso de las grandes empresas financieras con sus stakeholders, con el medioambiente y con la sociedad en general.

Palabras clave: responsabilidad social corporativa, sostenibilidad, inversiones socialmente responsables, desempeño financiero, solvencia bancaria.

\title{
Corporate Social Responsibility in Spanish Financial Institutions
}

\begin{abstract}
This research aims to analyze the responsible practices developed by financial institutions, as related to corporate social responsibility (CSR), and determine if the degree of intensity in the execution of these responsible policies is related to the size or solvency of these institutions.

On the basis of a positivist approach and using the main measurement standards of CSR, the validation of some hypotheses based on a previous empirical analysis was carried out. A sample of financial institutions was used to understand the degree of commitment of large financial companies to their stakeholders, the environment, and society in general.
\end{abstract}

Keywords: corporate social responsibility, sustainability, socially responsible investments, financial performance, bank solvency. 


\section{Introducción}

La 'responsabilidad social corporativa' (RSC), también conocida como 'responsabilidad social empresarial' (RSE), es un concepto sobre el cual los investigadores no comparten una definición común, a pesar de tratarse de un tema que lleva estudiándose desde hace varias décadas (Crane et ál., 2008). Además, la Rsc encuentra ubicación en diferentes disciplinas dentro del área de los negocios, como, por ejemplo, dirección estratégica, finanzas, ética o recursos humanos, entre otras (García-Santos y MaderoGómez, 2016), lo que implica tomar en consideración diferentes enfoques y posiciones ideológicas.

De esta manera, la RSC se considera una planificación de gestión empresarial que consiste en el análisis del impacto que provoca su actividad sobre el medioambiente, accionistas, consumidores, trabajadores y la sociedad en general. Por tanto, esta planificación estratégica conlleva a ejecutar de manera ineludible la legislación existente en el ámbito social, medioambiental, laboral y de derechos humanos. Además, también implica que la organización pueda realizar cualquier acto que desee siempre que favorezca la calidad de vida de sus empleados y del conjunto de la sociedad (Observatorio de Responsabilidad Social Corporativa, 2014). Así pues, es ampliamente aceptado que, mediante la Rsc, las empresas incorporen voluntariamente, en su actividad comercial y en sus relaciones con los stakeholders, las preocupaciones que presenta la sociedad en materia económica y medioambiental (Castro, 2010). La gestión de la imagen de la empresa ha adquirido, pues, una gran relevancia para las corporaciones modernas y se convierte en un instrumento de diferenciación y obtención de ventajas competitivas (Balmer, 2008).

En este sentido, el estudio de la imagen corporativa es considerado de gran relevancia y tiene el fin de relacionar lo que se quiere transmitir al público y a los stakeholders, y lo que realmente estos perciben. Asimismo, su importancia incluye el estudio de la imagen corporativa de la empresa para diferenciarse con la competencia y recuperar la confianza de los consumidores en la eficiencia de los negocios tras el impacto de la crisis financiera. 
Por consiguiente, las estrategias que ejecutan las entidades financieras están determinadas por la respuesta de los clientes a los modelos RSC. Así, la imagen positiva que ofrece la aplicación de estos modelos Rsc a las empresas puede asociarse con que las empresas logren establecer nuevas oportunidades de negocio, reduzcan su grado de riesgo operativo en un mundo globalizado o gestionen de una forma más avanzada los recursos humanos. Sin embargo, la vertiente negativa de estos modelos implica que la empresa reciba críticas por considerar que sus acciones están relacionadas con la moda, con la imagen empresarial o simplemente que suponga una distracción de su actividad principal, perjudicándolo cual perjudica, por ejemplo, a sus accionistas. Ante esta disyuntiva, se han realizado algunos estudios para conocer las principales ventajas que perciben los directivos sobre la función de responsabilidad social (KPMG, 2011; Fundación Adecco, 2012).

Los resultados de un estudio realizado con una muestra de 250 directores de recursos humanos de España, seleccionados por la fundación Adecco, señalan como ventaja fundamental que la relación con los stakeholders y la cohesión interna con sus empleados mejora. Sin embargo, establecen en tercera posición el afianzamiento de la imagen externa corporativa (figura 1).

Figura 1. Beneficios de la RSC

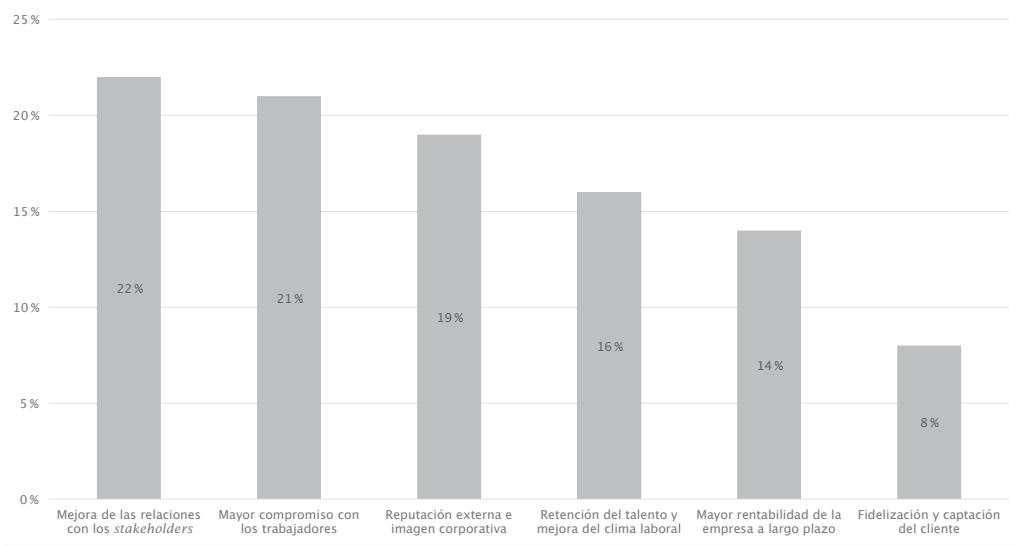

Fuente: Fundación Adecco (2012). 
Por su parte, KPMG, a partir de una muestra de 340 empresas de todo el mundo, concluyó que estas consideran más relevantes la reputación o marca de la organización, las consideraciones éticas y, en tercer lugar, la motivación de sus empleados (figura 2).

Figura 2. Beneficios de la RSC

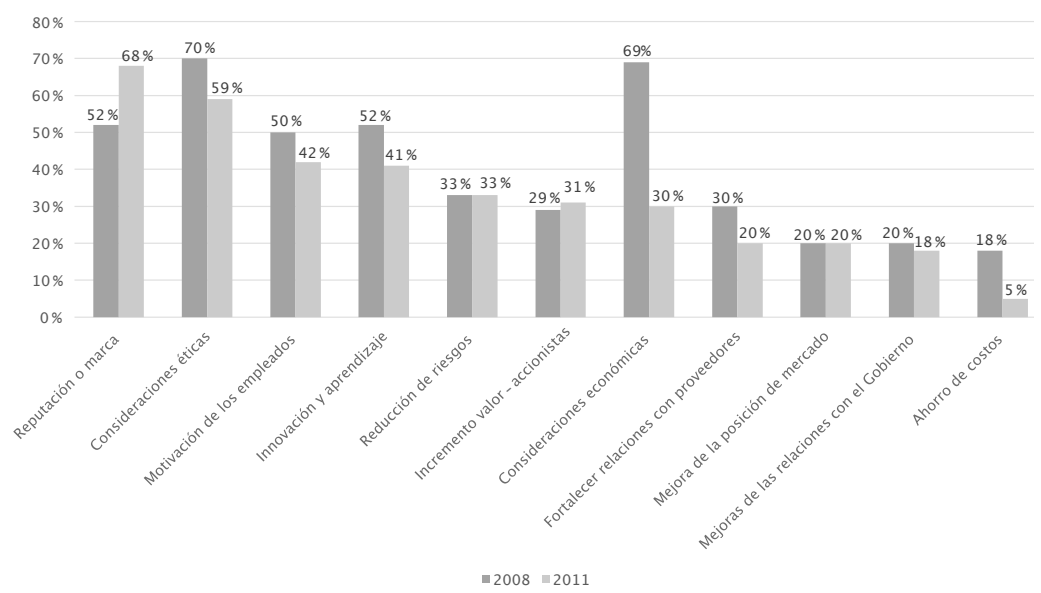

Fuente: KPMG (2009)

Las entidades financieras, para gestionar la comunicación de las acciones responsables que llevan a cabo, deben transmitir el verdadero valor creado con ellas. Por este motivo, la responsabilidad social se convierte en un aspecto que debe integrarse en los valores de la empresa, con el objeto de definir la imagen de sus marcas (Casaldáliga y Conde, 2012). Esta es una nueva exigencia en el mundo empresarial, por lo que es clave incluir la estrategia de responsabilidad desde la alta dirección que se encarga de liderar el proceso y transmitir los valores e intenciones a sus empleados para que puedan interiorizarlos. 


\section{Antecedentes}

\section{Origen y evolución de la responsabilidad social corporativa}

La responsabilidad social corporativa nace en Estados Unidos a finales de los años cincuenta. En esta etapa, la sociedad comienza a comprender la repercusión y el impacto de las organizaciones en el entorno que las rodea, pues considera que el empleo y la adquisición de factores productivos en estas organizaciones contribuyen al sostenimiento de la actividad económica. De esta manera, el concepto de RSC surge por primera vez en el año 1953, con Howard R. Bowen, economista americano considerado como el padre de la RSC. Bowen, en su obra Social responsabilities of the businessmen, apelaba a la responsabilidad social de las corporaciones para producir bienes y servicios, y devolver a la sociedad parte de lo que esta le había dado (Bowen, 1953). En una línea de pensamiento similar, Frederick (1960) hace hincapié en la necesidad de un uso social y público de los recursos de las empresas. Sin embargo, las contribuciones más relevantes a este concepto surgieron a partir de 1970, cuando se desarrollaron las diferentes teorías de la RSC. En las décadas de los setenta y ochenta, Sethi (1975), Carroll (1979) y Drucker (1984) abordan el aspecto ético de la RSC y la oportunidad de combinar una capacidad de producción por parte de las empresas con la satisfacción de necesidades humanas en las comunidades donde se opera. Así, evoluciona la idea de que sociedad y empresas deben conformar un conjunto inseparable y caminar en el mismo sentido (Wood, 1991).

La responsabilidad social de la empresa, en sentido estricto, no llega a España sino desde finales de los años 90, cuando la Asociación de Instituciones de Inversión Colectiva y Fondos de Pensiones (Inverco) introduce el concepto de 'inversión social responsable, siguiendo las tendencias mundiales y la creciente internacionalización económica (Anson, 2007).

Actualmente, la RSc es concebida como una estrategia de management que va más allá de los requerimientos mínimos que, en relación con la responsabilidad social, establecen las normas legales (McWilliams y Siegel, 
2001); es un compromiso por mejorar el bienestar de la comunidad a través de negocios sostenibles que beneficien a todos los stakeholders implicados (Kotler y Lee, 2005).

Por tanto, la RSC no es algo novedoso en el mundo empresarial, a pesar de que en esta última década esté alcanzando un mayor impacto, debido a que la mayoría de las grandes organizaciones fomenta acciones relacionadas con la RSC con sus principales stakeholders, como los trabajadores, proveedores y clientes. Toda empresa debe generar beneficios a través de su actividad empresarial, aunque no debe conformarse solo con estos beneficios económicos, ya que debe ir más allá hasta lograr una riqueza social a través de la incorporación voluntaria de preocupaciones sociales y medioambientales en sus actividades comerciales y en sus relaciones con los stakeholders.

\section{Relevancia de la información no financiera}

La creciente demanda por información global ha hecho que la importancia de los estados financieros, como vehículos de información hacia el exterior, haya perdido fuerza. En su lugar, el mercado está solicitando información sobre aspectos sociales y medioambientales. En este escenario, el informe integrado aparece como un actor relevante, dado que, en un solo documento, engloba información financiera y no financiera, con lo que muestra una imagen global de la compañía que informa, y hace énfasis en aspectos estratégicos, de gobierno y creación de valor (Navarrete y Gallizo, 2019).

Las empresas transforman sus prácticas empresariales y modelos de negocio a través de comportamientos sostenibles a largo plazo que puedan enfrentarse con un entorno que se someta a un continuo cambio debido a las nuevos paradigmas e innovaciones (Rivera-Arrubla et ál., 2016). Para conseguirlo plantean el diseño de nuevas estrategias, debido a que la adaptación a los nuevos tiempos implica la creación de canales alternativos de comunicación y formas de reporting más transparentes e inclusivas, de modo que puedan responder a una sociedad basada en la información (Soyka, 2013). De esta 
manera, las variaciones en las prácticas y en la presentación de informes han sido motivadas por las distintas partes interesadas, ya que demandan informes que permitan una rendición de cuentas más transparente.

Así pues, actualmente, clientes, consumidores, empleados, proveedores, organizaciones no gubernamentales y organismos privados y profesionales, entre otros, forman parte del público destinatario de la información y son los que se ocupan de presionar activamente para que los procesos de rendición de cuentas vayan más allá de las necesidades de los proveedores de capital financiero, a quienes los informes tradicionales atendían de manera prioritaria y exclusiva (Rivera-Arrubla et ál., 2016).

Además de la presión ejercida por los interesados directos en la información corporativa, algunos académicos e instituciones de estandarización no estatal, como el American Institute of Certified Public Accountants (AICPA), el Global Reporting Initiative (GRI), el Proyecto de Contabilidad Sostenible (A4S) y la International Federation of Accountants (IFAC), se han vinculado a este reclamo por informes más completos e incluyentes, con llamamientos que ratifican la necesidad creciente de una integración de los informes a distintos niveles: procesos organizacionales, toma de decisiones y presentación (Haller y Van Staden, 2014; Rivera-Arrubla et ál., 2016).

De esta manera, según The International Framework (2014), los objetivos del informe integrado son los siguientes:

- Mejorar la calidad de información disponible para permitir que los proveedores de capital financiero tengan una eficiente y productiva asignación de capital.

- Promover un enfoque más coherente y eficiente para que el reporting corporativo contribuya con diferentes informes estandarizados y comuniquen el rango completo de factores que materialmente afecten la habilidad de crear valor a lo largo del tiempo en una organización. 
- Mejorar la rendición de cuentas y administración sobre la amplia base de capitales (financiero, industrial, intelectual, humano, natural, social y relacional) y promover el entendimiento de sus interdependencias.

- Apoyar el pensamiento integrado, la toma de decisiones y acciones que se enfoquen en la creación de valor en el corto, medio y largo plazo. (The International Framework, 2014)

Las entidades financieras están obligadas a emitir dicho informe, siempre que cumplan determinadas condiciones de carácter financiero y de tamaño de la entidad (Ernst \& Young Global Limited, 2018). Así pues, algunas de las entidades financieras españolas que están obligadas a acogerse a los criterios que exigen el informe integrado son BBVA (2018) y Banco Santander (2018).

En definitiva, el Marco de reporte integrado publicado por el International Integrated Reporting Council (IIRC) proporciona a las organizaciones un marco para informar de manera integrada sobre asuntos como su entorno, modelo de negocio, estrategia, desempeño y perspectivas de futuro. Con este fin, se consideran tanto los aspectos financieros como los no financieros clasificados en seis formas diferentes de capital: financiero, industrial, humano, intelectual, social y natural. Este marco está diseñado para proporcionar a los inversores y otros grupos de interés información sobre cómo una organización crea valor en diversos ámbitos, como el social, el medioambiental y el económico. La aplicación de los principios del Marco hace que un informe de gestión sea más significativo (Ernst \& Young Global Limited, 2018).

\section{Normativa y estándares en Europa}

Los gobiernos y organizaciones internacionales han tomado iniciativas para aumentar la transparencia, promover las prácticas de buen gobierno, establecer principios y códigos de conducta con el fin de estimular y orientar a las empresas en la adopción de criterios de RSc. Paralelamente, han 
proliferado las empresas consultoras dedicadas a la definición de normas y de procedimientos para la elaboración de informes que faciliten la verificación de los resultados conseguidos en materia de RSC.

Las políticas de RSC son de implantación voluntaria en empresas e instituciones, de modo que no existe una legislación que regule este tipo de iniciativas privadas o públicas. A pesar de esta ausencia de regulación en las acciones socialmente responsables, los gobiernos y organizaciones internacionales han tomado iniciativas para aumentar la transparencia, promover las prácticas de buen gobierno, establecer principios y códigos de conducta con el fin de estimular y orientar a las empresas en la adopción de criterios de RSC. De esta manera, se han establecido unos estándares que hacen comparable la información divulgada por las distintas empresas en sus memorias. Estos estándares están recogidos en varias normas que han sido ampliamente estudiadas en la literatura empírica existente y de las que podemos destacar las siguientes:

- Accountability AA1000

- SA 8000

- Global Reporting Initiative (GRI) Standards

- Iso 26000

- ISO 9001 y 14001

Accountability (AA1000) es una red global sin ánimo de lucro, creada en 1995 para promover innovaciones que fomenten el desarrollo sostenible (Morrós y Vidal, 2005). Por su parte, la norma SA 8000 surgió en 1997, en Estados Unidos, con la misión de mejorar las condiciones de trabajo a nivel mundial; tiene por objetivo definir un conjunto de normas generales y auditables por una entidad externa sobre la protección de los derechos de los trabajadores (Heras Saizarbitoria y Arana Landín, 2013). La principal iniciativa para establecer un modelo estandarizado de aceptación internacional fue adelantada en 1997 por la Coalition for Environmentally Responsable Economies (CEREs) y el Programa Medioambiental de Naciones Unidas a través del GRI. Esta es una organización sin ánimo de lucro que 
promueve la sostenibilidad económica, ambiental y social. Además, ofrece a todas las empresas y organizaciones un marco de informes de sostenibilidad integral que es ampliamente utilizado en todo el mundo (Rodríguez y Ríos-Osorio, 2016).

Dentro de la familia de las normas Iso, se destaca especialmente la norma Iso 26000: 2010 en materia de responsabilidad social empresarial, puesto que esta norma proporciona una orientación para la gestión responsable de las empresas y no una lista de requisitos que deben cumplirse para obtener una certificación, como otras normas Iso (Organización Internacional de Normalización, 2010). Asimismo, la Iso 9001 es considerada de gran relevancia, ya que es una norma internacional que se aplica a los sistemas de gestión de calidad y que se centra en todos los elementos de administración de calidad con los que una empresa debe contar para tener un sistema efectivo que le permita administrar y mejorar la calidad de sus productos o servicios (Franch León y Guerra Bretaña, 2016).

Finalmente, la norma Iso 14001 tiene como finalidad colaborar con las organizaciones que estén interesadas en conseguir y colaborar con una adecuada actuación ambiental. En este sentido, las organizaciones interesadas en alcanzar sus objetivos medioambientales podrán valerse del modelo de gestión que presenta Iso 14001, integrándolo con otros requisitos de gestión como la norma Iso 9001, (Asociación Española para la Calidad, 2004).

En España, se dispone de abundante información sobre el desarrollo de la RSC. Las empresas consultoras, las fundaciones y las organizaciones interesadas en la difusión de la RSC publican regularmente informes sobre su grado de implantación. Allí suelen evaluar el tipo de Rsc que practican las empresas españolas analizando la información que presentan en sus memorias anuales o páginas web. Con diferentes criterios estudian sus contenidos y el grado de cumplimiento de los estándares más difundidos, como el GRI y el AA1000, entre otros (Fundación Ecología y Desarrollo [FED], 2005; KPMG, 2017). Otros informes valoran la situación de la RSC en España a partir de información recogida mediante encuestas de opinión realizadas a directivos (Deloitte, 2012; PWC, 2019) o consumidores (Forética, 2018). 
En estos informes se establecen escalafones, se identifican buenas prácticas y se proponen nuevas normas en el ámbito de la RSC.

\section{Bases teóricas: las teorías de la RSC}

Bajo el concepto de RSC se manifiesta un concepto común de empresa opuesto al enfoque contractual tradicional, en el que la empresa no se considera un ente abstracto, sino una institución social, coalición o asociación de agentes económicos aparte de los individuos que lo componen, con derechos y obligaciones (Rodríguez, 2003). Así pues, el campo de la responsabilidad social corporativa ofrece el desarrollo de determinadas teorías, así como una proliferación de enfoques, todos ellos controvertidos, complejos y poco definidos. Con el objeto de exponer su clasificación, asumimos las hipótesis de que estas teorías y enfoques de Rsc más relevantes muestran cuatro dimensiones relacionadas con los beneficios, la actuación política, las demandas sociales y los valores éticos. Estas hipótesis permiten estructurar las teorías de RSC en cuatro grupos:

\section{Teoría del accionista (stakeholder theory)}

Es ampliamente aceptado que la gestión de las organizaciones debe realizarse de manera que se cree el mayor valor posible para el accionista (Argandoña, 2007). En consecuencia, las organizaciones deberán tener como objetivo único maximizar beneficios económicos, siempre que actúen respetando las leyes y las costumbres éticas que rigen la sociedad (Friedman, 1962). En consecuencia, las acciones efectuadas por el gobierno corporativo desde un punto de vista responsable deberán atender a la preocupación constante de obtener beneficios de carácter puramente económico y de la creación del mayor valor posible para sus accionistas.

La responsabilidad social corporativa supone que las empresas no se pueden limitar a posicionarse entre sus competidores sin considerar la generación de un beneficio social y la creación de valor para el negocio. 
Este objetivo puede alcanzarse a través del desarrollo de nuevos productos y mercados, de la delimitación de la productividad en la cadena de valor y de la creación de grupos locales en los que la empresa actúa (Porter y Kramer, 2011).

En conclusión, las organizaciones, para optimizar su valor en la sociedad en la que actúan, tienen que cumplir el objetivo de maximizar el valor del capital que suponen sus stakeholders.

\section{Teoría de los stakeholders o de los grupos de interés}

La teoría de los stakeholders fue desarrollada por Edward Freeman (1984). En ella se establece y se estructura el concepto de stakeholders enfocado al nuevo paradigma de empresa que exigen las innovadoras circunstancias del siglo Xxi (Fernández Fernández y Bajo Sanjuán, 2012; Bajo Sanjuán et ál., 2013).

El concepto de stakeholder puede ser establecido como cualquier individuo o grupo de interés que puede repercutir o ser influido por la consecución de los objetivos establecidos por la organización (Cueva Estrada et ál., 2015). En consecuencia, teóricamente, la RSc obedece a una demanda de la sociedad o de los grupos de interés. Así pues, las expectativas de los grupos de interés han cambiado, por lo que las empresas ante un entorno internacional carente de regulación y crecientes amenazas globales no pueden buscar únicamente la consecución de beneficios económicos. Del mismo modo, según la teoría de los grupos de interés, es necesario que la organización considere a todos los partícipes que influyen o son afectados por la actividad empresarial que consiste en la consecución de los objetivos de la compañía (Freeman, 1984; Cueto Cedillo y De la Cuesta, 2017). De esta manera, las empresas conseguirán mejores resultados si valoran las demandas de sus stakeholders, debido a que fomentarán las relaciones bilaterales con los grupos de interés y asimismo mejorarán la gestión de los grupos de interés multilaterales (Freeman y William, 1990; Centro Médico Imbanaco, 2018). 
De otra parte, las empresas mantienen una estrecha relación con la sociedad y sus operaciones y actividades influyen en inversores, empleados, consumidores, comunidades y medioambiente de diversas maneras (Kreng y Huang, 2011). Como consecuencia, las organizaciones podrán lograr un mejor posicionamiento, además de alcanzar importantes beneficios, siempre que formalicen sus objetivos desde un punto de vista estratégico, de manera que pueda beneficiar la relación que mantiene la empresa con sus grupos de interés (Garrigues Walker y Trullenque Sanjuan, 2008). Teniendo esto en cuenta, podemos decir que la responsabilidad social abarcaría las expectativas económicas, legales, éticas o discrecionales de la sociedad sobre la organización en un momento dado (Carroll, 2016).

Esta teoría se considera de especial importancia para aquellos dirigentes de las organizaciones que pretenden comprender de manera más exhaustiva algunos de los aspectos más relevantes de sus grupos de interés. Por consiguiente, los procesos, las estrategias y las relaciones tendrán que formularse teniendo en consideración los diferentes tipos de grupos de interés existentes (Fernández Fernández, 2016). Así pues, resulta de especial conveniencia establecer e institucionalizar cauces de diálogo transparentes con los distintos grupos de interés como condición previa para una gestión exitosa y, de esta manera, se beneficiará la sostenibilidad de la empresa a plazo medio-largo (Fernández Fernández y Bajo Sanjuán, 2012). Las empresas deben tener en cuenta criterios de Rsc en la toma de decisiones, con el objetivo de buscar un equilibrio entre los beneficios para la propia empresa y la adopción de criterios que minoren los efectos negativos al entorno externo.

\section{Teoría de la ciudadanía empresarial (corporate citizenship theory)}

El impulso de la RSC a comienzos del siglo xxI va ligado a los escándalos financieros que han dejado en entredicho las actuaciones de las empresas y ha provocado que pierdan credibilidad y confianza. A principios del siglo XXI, las organizaciones empresariales experimentaron una pérdida 
de credibilidad y confianza, debido a la creciente importancia que obtuvo la RSC y a los escándalos financieros surgidos en esa etapa, como los de Enron (2001) y WorldCom (2002; Bronchain, 2003; Mozas Moral y Puentes Poyatos, 2010; Cueto Cedillo y Cuesta González, 2017).

Por esta razón, junto a estos enfoques, actualmente se está observando una tendencia a utilizar la teoría de la ciudadanía empresarial (corporate citizenship) para justificar la Rsc. Esta teoría considera que la empresa es parte de la sociedad y ha de participar en la vida social contribuyendo en alguna medida y voluntariamente al desarrollo y bienestar de la sociedad más allá de la creación de riqueza (Melé, 2007). Como corporación pública tiene obligaciones sociales, como la equidad, justicia social y protección de los trabajadores (Letza et ál., 2004). En este sentido, las organizaciones tienen el compromiso de ser "buenas ciudadanas" y, por consiguiente, sus actos derivados de la gestión empresarial tendrán que colaborar de una manera socialmente responsable con el bienestar de las comunidades en las que actúan (Moral y Puentes-Poyatos, 2010; Cueto, 2014; Cueto y De La Cuesta, 2017).

En conclusión, la definición de una sociedad se genera a partir de las empresas, pues tienen obligaciones sociales y, por tanto, deben considerarse como parte de la sociedad, por lo que sus actuaciones deben contribuir, de forma socialmente responsable, al bienestar de las demás comunidades que integran la sociedad. De esta manera, surge un nuevo concepto, el de stakeholder society o 'sociedad de afectados' (Weiss-Belalcázar, 2003).

\section{Estándares de medición de la RSC}

La medición es una tarea fundamental para conocer el grado de cumplimiento de los objetivos empresariales. Las organizaciones tendrán que establecer sus objetivos en función de los resultados obtenidos en relación con la RSE y, en consecuencia, tendrán que realizar los ajustes esenciales para conseguir un mejor rendimiento (Duque Orozco y Cardona Acevedo, 2013). 
Un indicio de buen management y la consolidación de un campo de estudio se detecta a partir de las mejores prácticas de medición (Pfeffer, 1993). El campo de la RsC cumple con este precepto y, por consiguiente, se ha desarrollado una discusión sobre el contenido de la RSC y su medida (Perdomo y Escobar, 2011).

\section{Pacto Mundial de las Naciones Unidas (uN Global Compact)}

El Pacto Mundial de las Naciones Unidas es una iniciativa mundial cuya misión consiste en generar un movimiento internacional de empresas sostenibles. Así pues, el Pacto Mundial de Naciones Unidas es un llamado a las empresas y organizaciones a que alineen sus estrategias y operaciones con diez principios universales sobre derechos humanos, normas laborales, medioambiente y lucha contra la corrupción. Asimismo, cuenta con el mandato de la onu para promover los objetivos de desarrollo Sostenible en el sector privado (Pacto Mundial, 1999). Se trata de un contexto empírico que presenta a sus firmantes un gran conjunto de recursos que les facilita la realización de modelos de negocio y desarrollo sostenible, y permite fomentar, establecer y difundir políticas de sostenibilidad empresarial.

En la actualidad, hay más de 12500 organizaciones empresariales suscritas al Pacto Mundial. Entre estas empresas se encuentra la Red Española, la red local con mayor número de firmantes, considerada una de las primeras plataformas nacionales de este Pacto, debido a que su estructura organizativa y su modelo de gestión es de los más innovadores. De igual modo, cuenta con una destacada red local, a causa del tipo de actividades realizadas y de las herramientas empleadas, que le permite fomentar el establecimiento de los Diez Principios del Pacto Mundial, además de sensibilizar al sector privado para que coopere con los objetivos establecidos por la onU (Pacto Mundial, 1999). En concreto, la Red Española del Pacto Mundial cuenta en la actualidad con más de 1500 empresas firmantes, que representamos, clasificadas según su tamaño, en la figura 3. 
Figura 3. Red Española del Pacto Mundial

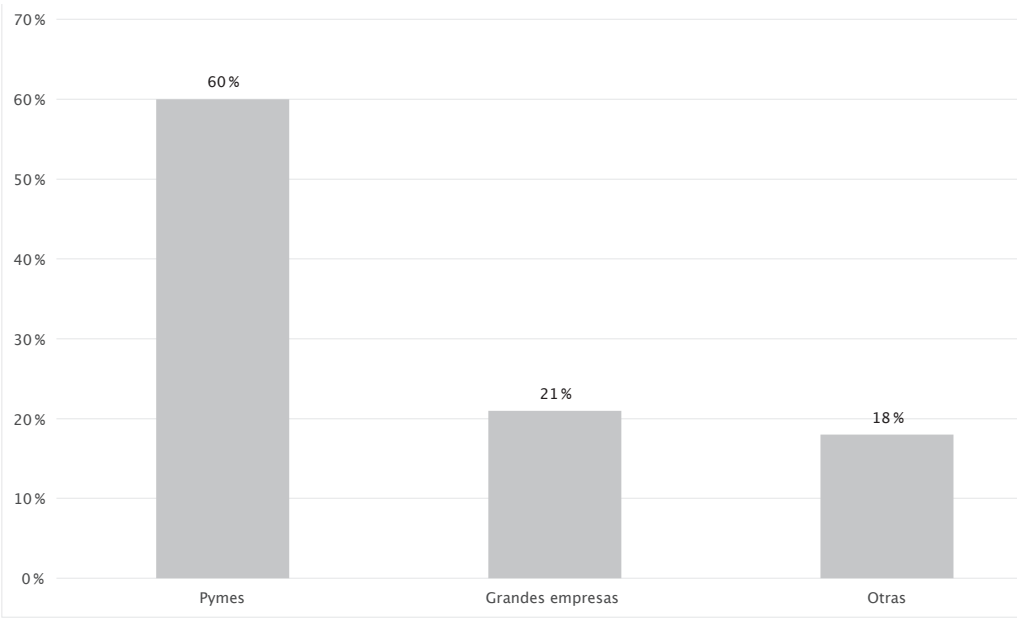

Fuente: elaboración propia.

Esta iniciativa persigue un doble objetivo, pues pretende que las empresas adopten un conjunto de principios universales en materia de derechos humanos, derechos laborales, medio ambiente y lucha contra la corrupción (Armijos-Buitrón, Paladines-Benítez y Costa-Ruiz, 2019). Igualmente, propone canalizar acciones en apoyo de los objetivos más amplios de las Naciones Unidas, donde se incluyen los objetivos de desarrollo sostenible (oDs) (Pacto Mundial, 1999).

Es ampliamente aceptado que el Pacto Mundial es un instrumento necesario en las organizaciones empresariales para conseguir un comportamiento responsable. A pesar de ello, los estudios empíricos publicados hasta el momento no han analizado el efecto de este instrumento en las actividades recientes desarrolladas por las empresas adheridas (Rodríguez et ál., 2019). 


\section{Global Reporting Initiative Standards}

La progresiva sensibilización de las organizaciones en materia de sostenibilidad ha incentivado la creación de ciertos indicadores de gestión que logren ser aceptados universalmente para que, así, pueda medirse el compromiso empresarial con la sociedad y el medio ambiente. Por esta razón, existe un constante interés en incentivar a las empresas a generar informes anuales basados en sostenibilidad. Además, debe considerarse la relevancia de la adopción del desarrollo de estos informes a las economías emergentes.

De esta manera, la RSC se enfoca en el logro de los objetivos estratégicos de la empresa, que deben ser paralelos a la forma de actuar de esta, ya que es primordial para el correcto funcionamiento de la empresa y, por tanto, su perdurabilidad en el tiempo. Por esto, desde el 2018 se obliga a las empresas a reportar sus informes de sostenibilidad, en los que se muestra el compromiso de estas con el medio ambiente además de mostrar sus resultados financieros. El modelo que recoge los principales aspectos por considerar para la elaboración de estos informes es justamente el GRI.

La GRI es un organismo multistakeholder independiente, que tiene como finalidad suministrar un marco eficaz para la proyección de memorias de sostenibilidad, de manera que las organizaciones, cualquiera que sea su tamaño, su sector o su ubicación, puedan valerse de él cuando lo estimen necesario (Global Reporting Initiative, 2002).

La GRI se constituyó en el año 1997 como una iniciativa conjunta de las organizaciones no gubernamentales estadounidenses Coalition for Environmentally Responsible Economies (Ceres) y el Programa de las Naciones Unidas para el Medio Ambiente (PNUMA). Tiene sede en Ámsterdam y actualmente en ella participan representantes de organizaciones de derechos humanos, derechos laborales, investigación, medioambientales, corporaciones, inversionistas y organizaciones contables de todo el mundo. El principal alcance de este organismo consiste en optimizar la calidad en el desarrollo de las memorias de sostenibilidad, con el objeto de confrontarlos con los informes financieros en lo que se refiere a 
comparabilidad, precisión, fiabilidad, regularidad y verificabilidad (Global Reporting Initiative, 2002).

La visión principal del GRI es conformar una comunidad global próspera que fomente la progresión de la humanidad y mejore los recursos de los que depende. Por su parte, la misión principal es empoderar decisiones que generen beneficios sociales, ambientales y económicos para todos.

Los estándares GRI son considerados las mejores prácticas a nivel global para informar públicamente los impactos económicos, ambientales y sociales de una organización. De esta forma, la elaboración de informes de sostenibilidad a partir de estos estándares facilita información sobre las contribuciones positivas y negativas de las empresas al desarrollo sostenible. Los estándares GRI están principalmente destinados a ser usados a la hora de elaborar informes de sostenibilidad que se centran en temas materiales. Desde la creación de estos estándares, se ha difundido su adaptación a diferentes organizaciones y, en la actualidad, son frecuentemente adoptados por gran parte de las organizaciones (Global Reporting Initiative, 2016). Igualmente, la GRI es considerada el marco más popular para el reporte de RSC, debido a que el $93 \%$ de las 250 empresas más grandes del mundo realizan informes sobre su desempeño en materia de sostenibilidad aplicando los estándares GRI (KPMG, 2017).

Con el objetivo de llevar a cabo su misión, la GRI ha creado cuatro áreas de enfoque para los próximos años:

1. Crear normas y una guía para avanzar con el desarrollo sostenible.

2. Armonizar el panorama de sostenibilidad.

3. Liderar informes de sostenibilidad eficientes y efectivos.

4. Impulsar el uso efectivo de la información de sostenibilidad para mejorar el rendimiento. 


\section{ISO 26000}

La responsabilidad social, de acuerdo con esta norma, se define como la responsabilidad de una organización por los impactos de sus decisiones y actividades, en la sociedad y en el medio ambiente, a través de una conducta ética que debe cumplir con el desarrollo sostenible y estar integrada en la totalidad de la organización y puesta en práctica en todas sus relaciones (Montero, 2012). La norma Iso 26000 tiende a convertirse en una poderosa herramienta para ayudar a las empresas, gobiernos, asociaciones, entidades y organizaciones no gubernamentales para incorporar la responsabilidad social como un principio de buena gestión, de modo que contribuye a un uso más consciente y sostenible de los recursos naturales y humanos (Puterman, 2010). Por esta razón, se considera que la norma ofrece una guía global sobre RSC tanto para empresas del sector público como del sector privado, puesto que suministra, por su continua y exitosa utilización, reglas, líneas directrices o resultados que garantizan un nivel de orden óptimo en un determinado contexto (Otxoa-Errarte y Landa, 2012). En consecuencia, es ampliamente aceptado que la norma iso 26000 incentiva la aplicación de mejores prácticas de RSC en todas las organizaciones a nivel mundial.

Esta norma extiende la comprensión e implantación de la responsabilidad social mediante los siguientes procesos:

- Desarrollo de consenso internacional sobre el significado de la Rsc y los asuntos relacionados a los que las empresas necesitan hacer frente.

- El aporte de una guía para la traducción de los principios en acciones efectivas.

- La afinación de las mejores y más actuales prácticas, y la difusión de la información para el bien de la comunidad internacional.

La puesta en funcionamiento de esta norma en las diferentes organizaciones ofrece cada vez más importancia en el ámbito empresarial, debido a que además de mostrar un buen servicio al cliente sin dañar el medioambiente, ofrece a su vez una forma de operar socialmente responsable. Sin embargo, a pesar de su gran alcance, la aplicación de la Iso 26000 
es voluntaria (Montero, 2012), al contrario de lo que ocurre con la Iso 9001 y la Iso 14001, que son de obligatorio cumplimiento (Tarí et ál., 2012).

Esta norma tiene siete materias fundamentales sobre las que se proyecta la actuación empresarial responsable: gobernanza de la organización, derechos humanos, prácticas laborales, medio ambiente, prácticas justas de operación, consumidores y participación y desarrollo de la comunidad. Cada una de estas materias fundamentales incluye una serie de asuntos junto con la propuesta de acciones y expectativas relacionadas. Así, por ejemplo, en materia de medio ambiente se describen cuatro asuntos: la prevención de la contaminación, el uso sostenible de los recursos, la mitigación del cambio climático y la adaptación a este, la protección del medio ambiente, la biodiversidad y restauración de los hábitats naturales (Otxoa-Errarte y Landa-Oñati, 2012).

\section{Informe de Sostenibilidad}

Desde los años 70 hay un interés creciente por parte de las empresas en informar sobre cuestiones de RSC (Zorio-Grima et ál., 2015). Esta tendencia se originó entre las grandes firmas multinacionales, de manera que cada vez son más las organizaciones que tienen conciencia de ella y publican informes de manera voluntaria (KPMG, 2011). Así pues, el Informe de sostenibilidad constituye un instrumento útil para la gestión empresarial (Palacios y Coppa, 2015).

A pesar de que existen distintas normas y directrices sobre cómo elaborar un informe de RSC (OCDE, Global Compact y Pacto Mundial, entre otras), la mayoría de dichos informes se confeccionan siguiendo lo establecido por el Global Reporting Initiative (Zorio-Grima et ál., 2015).

De otra parte, en el ámbito internacional existen dos índices que miden, además de la rentabilidad de las empresas según su cotización en bolsa, el nivel de prácticas de RSC aplicadas a sus planes de gestión. Estos índices son el FTSE4Good y el Dow Jones Sustainability Index (DJSI). 
El índice FTSE4Good, desarrollado por FTSE Russell, evalúa el rendimiento de las empresas sobre la base de su compromiso por integrar prácticas que tengan en consideración criterios medioambientales, sociales y de gobernanza empresarial, para generar rendimientos financieros competitivos a largo plazo e impacto social positivo. Estas prácticas reciben el nombre de ESG (environmental, social and governance) (Fundación Corresponsables, 2019).

Por otro lado, el Dow Jones Sustainability Index utiliza un conjunto de índices de sostenibilidad que integran a las compañías cotizadas en la bolsa y con alto desempeño en materia de sostenibilidad, es decir, aquellas que cumplen con los criterios ESG (Pincheiro et ál., 2018).

Este índice se elabora en base a criterios de desempeño económico, ambiental y social de la empresa, evaluando temas como el gobierno corporativo, la gestión de riesgos, la marca, cambio climático, estándares de la cadena de suministro y prácticas laborales. La tendencia es rechazar las empresas que no operan de manera sostenible y ética (Forcadell y Aracil, 2017).

\section{Libro verde de la Comisión Europea sobre RSC}

El Libro verde de la Comisión Europea sobre Rsc (Comisión de las Comunidades Europeas, 2001) es un documento de carácter no vinculante en el que se incluyen recomendaciones acerca de comportamientos considerados idóneos en materia de RSC, así como una serie de principios básicos encomendados a las empresas. Este carácter implica que no sea posible una medición cuantitativa acerca del cumplimiento, por parte de las empresas, de las recomendaciones allí expresadas.

El documento tiene como objetivo complementar y mejorar las acciones ya existentes en materia de RSC para lo que elabora principios, enfoques e instrumentos para la promoción de buenas prácticas e ideas innovadoras. Con el Libro verde se inicia un amplio debate de ideas sobre Rsc a nivel 
nacional e internacional. Supone el compromiso de la UE con la RSC y promueve los comportamientos socialmente responsables en la búsqueda de objetivos de crecimiento económico, competitividad, justicia social y desarrollo sostenido (Pinillos y Fernández, 2011).

Posteriormente, la Comisión elabora una nueva comunicación sobre RSC (Comisión de las Comunidades Europeas, 2002). En ella se profundiza y se completan las ideas del Libro verde (Elósegui, 2010). En relación con las entidades financieras, la Comisión elaboró el Libro verde, sobre los servicios financieros al por menor (Comisión de las Comunidades Europeas, 2015), que tiene como objetivo el debate sobre la apertura del mercado europeo de servicios financieros al por menor. Busca obtener una adecuada protección de los consumidores y mejores resultados tanto para ellos como para las empresas financieras.

Por su parte, la Confederación Española de Cajas de Ahorros (CECA) ha analizado las prácticas vinculadas al negocio financiero y a la RSC de sus empresas, comparándolas con un conjunto de bancos del resto del mundo. En sus conclusiones se destaca el alto compromiso de este tipo de entidades financieras españolas en lo referente a responsabilidad social y sostenibilidad (Responsables Consulting, 2008).

\section{Recolección de datos y procesamiento}

Como ya se indicó anteriormente, el principal objetivo de este estudio consiste en analizar una muestra de las entidades financieras españolas para comprobar si el sector financiero español cumple con los principios y recomendaciones promulgados en el Libro verde de responsabilidad social corporativa, es decir, con los estándares de medición de la RSC antes mencionados y, complementariamente, determinar si el tamaño o el nivel de solvencia de la entidad están relacionados con el nivel de cumplimiento de esos principios de responsabilidad. 
Dado el elevado número de entidades financieras existentes en España, así como la diversidad de estas, hemos fijado un criterio de selección, en la línea establecida en diversos trabajos empíricos previos (Benito y Bastida, 2007; Navarro et ál., 2008; Pina et ál., 2009).

De esta forma, para realizar la investigación, se ha escogido una muestra compuesta por las 32 entidades financieras españolas más solventes, según las valoraciones otorgadas por las agencias de calificación de riesgo Fitch, Moody's y Standard \& Poor's. A las calificaciones crediticias a corto y largo plazo concedidas por cada agencia de calificación de riesgo (afectadas por la perspectiva de la calificación, en su caso) se le ha asignado un valor numérico, el cual, agregado, ha dado como resultado el valor de la variable Solv (solvencia).

La información acerca del cumplimiento de los estándares de aplicación de RSC se ha obtenido directamente de las entidades financieras objeto de estudio y publicada en sus páginas web corporativas. Así pues, este trabajo tiene un alto carácter descriptivo, y plantea un análisis de tipo cualitativo. De acuerdo con investigaciones anteriores, en materia de tratamiento de información de este tipo, se ha realizado un diseño transversal y no experimental, sin intromisión en los resultados publicados por las empresas (Huang y Chao, 2001; Benito y Bastida, 2007; Rodríguez et ál., 2007).

Los datos utilizados para el análisis son los recogidos en los informes anuales de las empresas para el año 2018, de modo que se trata de la información más reciente.

Por su parte, las variables que han medido el tamaño de la entidad han sido dos: tamaño de los activos y nivel de resultados, ambos en el ejercicio económico de referencia. 


\section{Resultados empíricos}

Para poder obtener conclusiones acerca de las relaciones entre el grado de compromiso, en materia de RSC, de las entidades financieras y su tamaño y nivel de solvencia, hemos procedido a clasificar las entidades objeto de estudio según los siguientes criterios: aplicación de estándares de RSC (RSC-St), tamaño de activos (Asset-Sz), nivel de beneficios (Perf) y nivel de solvencia (Solv), mediante la aplicación de un criterio ordinal para su clasificación; de esta manera, obtienen un mayor valor aquellos ítems que indican un mejor posicionamiento en su respectivo escalafón. Este procedimiento jerárquico ya ha sido utilizado en la literatura previa por Navarro et ál. (2010) al analizar la implantación de Rsc en organismos locales.

Aplicando la metodología antes citada, hemos tomado la puntuación obtenida por cada entidad y hemos asignado la puntuación a cada nivel de estudio. Esto nos ha permitido elaborar rankings en función de cada una de las variables por estudiar.

\section{Establecimiento de hipótesis}

Una vez que la información disponible se adapta metodológicamente a nuestro objeto de estudio procedemos a plantear las siguientes hipótesis.

\section{Hipótesis general}

Las entidades financieras de mayor tamaño y las más solventes aplican en mayor medida políticas responsables

De esta hipótesis general se desprenden las siguientes hipótesis específicas:

- En relación con las variables que miden el tamaño de la entidad: 
H1: el nivel de aplicación de estándares de RSC por parte de las entidades financieras está directa y positivamente relacionado con el tamaño de la entidad.

Esta, a su vez, puede dividirse en dos subhipótesis:

H1a: el nivel de aplicación de estándares de RSC (RSC-St) por parte de las entidades financieras está directa y positivamente relacionado con el tamaño de sus activos (Asset-Sz).

Parece razonable suponer que las entidades con un mayor volumen de activos (mayor dimensión) serán más propensas a invertir (destinar recursos) a políticas de RSC.

H1b: el nivel de aplicación de estándares de RSC (RSC-St) por parte de las entidades financieras está directa y positivamente relacionado con su volumen de beneficios (Perf).

Aquellas entidades que obtienen un mayor volumen de beneficios podrán destinar, con mayor facilidad, una parte de aquellos a políticas de RSC.

- En lo que respecta a la relación entre estándares de RSC y solvencia, nos lleva a plantear la siguiente hipótesis:

H2: el nivel de aplicación de estándares de RSC (RSC-St) por parte de las entidades financieras está directa y positivamente relacionado con el nivel de solvencia (Solv).

Un mejor nivel de solvencia alienta a la entidad a destinar recursos, en mayor medida, a políticas de Rsc.

En consonancia con el tamaño muestral utilizado, la metodología utilizada para testar estas hipótesis ha sido el coeficiente de correlación de Pearson con prueba de significación bilateral, usando el software estadístico sPss Statistics 20. Además, al tratarse de un tamaño muestral relativamente pequeño, se exigirá un elevado nivel de significación en los resultados, 
para descartar la casualidad en los mismos. Por tanto, para admitir que una correlación entre variables es estadísticamente significativa deberán producirse correlaciones elevadas y con un nivel de confianza (probabilidad de error) del $1 \%$.

Los resultados obtenidos (tabla 1) han sido los siguientes:

Tabla 1. Correlaciones

\begin{tabular}{|l|l|c|c|c|c|}
\cline { 3 - 6 } \multicolumn{2}{c|}{} & RSC-St & Asset-Sz & Perf & Solv \\
\hline \multirow{3}{*}{ RSC-St } & Correlación de Pearson & 1 &, $777^{* *}$ &, $894^{* *}$ &,- 156 \\
\cline { 2 - 6 } & \multirow{3}{*}{ Sig. (bilateral) } & &, 001 &, 000 &, 060 \\
\cline { 3 - 6 } & & 32 & 32 & 32 & 32 \\
\hline
\end{tabular}

\begin{tabular}{|c|c|c|c|c|c|}
\hline & \\
\hline & & RSC-St & Asset-Sz & Perf & Solv \\
\hline \multirow{3}{*}{ Asset-Sz } & Correlación de Pearson &, $777^{\star \star}$ & 1 &, $923^{\star *}$ &,- 163 \\
\hline & \multirow{2}{*}{ Sig. (bilateral) } &, 001 & &, 000 & ,612 \\
\hline & & 32 & 32 & 32 & 32 \\
\hline \multirow{3}{*}{ Perf } & Correlación de Pearson &, $894^{* *}$ &, $923^{* *}$ & 1 &,- 268 \\
\hline & \multirow{2}{*}{ Sig. (bilateral) } &, 000 &, 000 & & ,399 \\
\hline & & 32 & 32 & 32 & 32 \\
\hline \multirow{3}{*}{ Solv } & Correlación de Pearson &,- 156 &,- 163 &,- 268 & 1 \\
\hline & \multirow{2}{*}{ Sig. (bilateral) } &, 060 & 612 & 399 & \\
\hline & & 32 & 32 & 32 & 32 \\
\hline
\end{tabular}




\section{Discusión de resultados}

Los resultados obtenidos han confirmado que el nivel de aplicación de los estándares de RSC por parte de las entidades financieras españolas está relacionado con el tamaño de estas (medido a través de sus activos). Los resultados de correlación $(0,777)$ y de significación, o probabilidad de error, del $1 \%$ confirman la existencia de una relación directa y positiva entrelas dos variables (RSC-St y Asset-Sz). Esto significa que, a medida que las entidades van creciendo, también lo hace su nivel de compromiso con las políticas de RSC, en consonancia con lo ya apuntado en la literatura por la Confederación Española de Cajas de Ahorros (Responsables Consulting, 2008).

Este estudio confirmó que el nivel de aplicación de estándares de RSC por parte de las entidades financieras españolas está directamente correlacionado y de forma positiva con su volumen de beneficios, con una correlación directa y positiva de 0,894 y una significación del $1 \%$. Estos resultados indican que aquellas entidades más rentables están dispuestas a utilizar un mayor volumen de recursos para políticas sostenibles, lo que indica un alto grado de compromiso social y confirma lo que anteriormente ya fue apuntado en el informe Adecco, en el que los directivos expresaban sus opiniones acerca de las ventajas de invertir en políticas de responsabilidad social (Adecco, 2012).

La hipótesis que establecía que el nivel de aplicación de estándares de RSC por parte de las entidades financieras está directa y positivamente relacionado con el nivel de solvencia no puede ser admitida, atendiendo a los resultados obtenidos en nuestro estudio, tanto por los bajos niveles de correlación como por la escasa significación de los resultados. Este dato abre el camino a nuevas perspectivas de investigación acerca de los motivos que pueden llevar a los gestores delas entidades más solventes a no ser las que más se involucran con la RSC. No obstante, a pesar de no verificarse esta última hipótesis, podemos deducir, indirectamente, la existencia de un elevado grado de implicación, por parte de las entidades estudiadas, con las recomendaciones establecidas tanto en el Libro verde de la Comisión 
de las Comunidades Europeas, como en la posterior Comunicación de la Comisión y en su adaptación, específica para el sector financiero (Comisión de las Comunidades Europeas, 2001; 2002; 2015).

\section{Conclusiones}

La responsabilidad social corporativa es una preocupación constante en el sector financiero, debido a que las empresas financieras son conscientes de la importancia de las prácticas responsables en los modelos de negocio. En este trabajo hemos expuesto las tendencias actuales en materia de RSC, analizando los principales estándares que, hoy día, se consideran fundamentales en una adecuada y completa política de RSC. No obstante, a pesar de la gran relevancia que estas actuaciones suponen, no son de aplicación obligatoria.

Así pues, en nuestros resultados hemos comprobado que todas las entidades financieras analizadas han puesto en práctica actuaciones referenciadas a la RSC en su actividad empresarial. Por el contrario, se ha evidenciado que, a pesar de la gran conciencia y la puesta en práctica de acciones socialmente responsables, no todas las empresas emiten sus informes de RSC por lo que se concluye que aún queda mucho camino por avanzar en este aspecto.

De otra parte, las inversiones socialmente responsables, con sus respectivos criterios ESG, cada vez poseen mayor importancia en lo que a materia de inversión se refiere. En general, las entidades financieras analizadas ofrecen cada vez más productos sostenibles que a su vez contribuirán a la responsabilidad medioambiental, económica y del propio gobierno corporativo.

Asimismo, la literatura empírica existente a nivel internacional defiende que las empresas con un mayor tamaño realizarán unas mejores estrategias de aplicación de Rsc en sus actividades. Por este motivo, hemos querido comprobar el nivel de implicación de las entidades financieras españolas con las políticas de Rsc. Mediante un estudio empírico, se ha analizado esta implicación y se ha comprobado las posibles relaciones entre el 
cumplimiento de los principales estándares de RSC y el tamaño o nivel de solvencia de las entidades financieras. A su vez, el tamaño de la entidad se ha considerado desde dos ópticas distintas, el volumen de sus activos y su volumen de beneficios.

Finalmente, esta parte empírica se ha podido concluir que tanto el volumen de los activos como el nivel de beneficios obtenidos están directa y positivamente relacionados con el cumplimiento de los principales estándares de RSC.

Por su parte, los resultados indican la inexistencia de una relación directa entre el grado de solvencia de las entidades financieras y el cumplimiento de estándares de Rsc. Es decir, un mayor nivel de solvencia en una entidad no conlleva, necesariamente, a mayores acciones socialmente responsables.

\section{Referencias}

Armijos-Buitrón, J. Paladines-Benítez y M. Costa-Ruiz (2019). El sector empresarial ecuatoriano frente a la Agenda 2030: desafíos y oportunidades. XIV Congreso Ibérico de Sistemas y Tecnologías de la Información (CISTI), Coimbra, Portugal, 1-6. DoI: 10.23919/CISTI.2019.8760799

Anson, R. (2007). Responsabilidad social de las empresas. Cuenta y Razón, 145, 11-15. Argandoña, A. (2007). Responsabilidad Social y creación de valor para el accionista. ¿Objetivos contrapuestos o complementarios? Cátedra "La Caixa" de Responsabilidad Social de la empresa y gobierno corporativo. [publicación ocasional, 07/16]. Consultado en https://www.fundacionseres.org/lists/informes/attachments/1101/responsabilidad\%20social\%20y\%20creacion $\% 20$ de\%20valor\%20para\%20el\%20accionista.pdf

Asociación Española para la Calidad. (2004). Norma UNE-es Iso 14001. AEC. Consultado en https://www.aec.es/web/guest/centro-conocimiento/ norma-une-en-iso-14001

Bajo Sanjuán, A., González, M. y Fernández, J. L. (2013). Responsabilidad social y empresa sostenible. AdComunica. Revista Cientifica de Estrategias, 
Responsabilidad social corporativa

Tendencias e Innovación en Comunicación, 5, 223-243. DoI: http://dx.doi. org/10.6035/2174-0992.2013.5.14

Balmer, J. (2008). Identity based views of the corporation: Insights from corporate identity, organisational identity, social identity, visual identity, corporate brands and corporate image. European Journal of Marketing, 42(9-10), 879906. DoI: https://www.doi.org/10.1108/03090560810891055

Banco Santander. (2018). Banca responsable. Estado de información no financiera consolidado. Consultado en https://www.santandersostenibilidad.es/recursos/ doc/portal/2018/05/15/informe-de-sostenibilidad-2018.pdf

BBVA. (2018). Estado de información no financiera. https://accionistaseinversores. bbva.com/microsites/cuentasanuales2018/informe-de-gestion/estado-deinformacion-no-financiera/index.html

Bronchain, P. (2003). Towards a sustainable corporate social responsibility. European Foundation for the Improvement of Living and Working Conditions.

Benito, B. y Bastida, F. (2007). Política y Gestión Financiera Municipal. Revista de Contabilidad - Spanish Accounting Review, 11(2), 43-66.

Bowen, H. F. (1953). Social responsibilities of the businessmen. Nueva York: Harper \& Row.

Carroll, A. B., (1979). A Three-Dimensional Conceptual Model of Corporate Social Performance. Academy of Management Review, 4, 497-505.

Carroll, A. B. (2016). Carroll's pyramid of csR: taking another look. International Journal of Corporate Social Responsibility, 1(3), 1-8.

Casaldáliga, N. y Conde, V. (2012). Mejorar la imagen de marca a través de la Responsabilidad Social Corporativa. Harvard Deusto Márketing y Ventas, 109, 34-42.

Castro, J. (2010). La responsabilidad social empresarial (RSE), un nuevo reto para las mipyme. Revista Activos, 15, 101-120.

Centro Médico Imbanaco. (2018). Memoria de Sostenibilidad Acciones que reflejan nuestro compromiso con la vida. Consultado en https://www.imbanaco.com/ wp-content/uploads/2019/06/SOSTENIBILIDAD-2018-CMI.pdf

Comisión de las Comunidades Europeas (2001). Libro verde. Fomentar un marco europeo para la responsabilidad social de las empresas. Obtenido de www. europarl.europa.eu/. 
Comisión de las Comunidades Europeas. (2002). Comunicación de la Comisión relativa a la responsabilidad social de las empresas: una contribución empresarial al desarrollo sostenible. Consultado en https://op.europa.eu/en/ publication-detail/-/publication/6e2c6d26-d1f6-48a3-9a78-f0ff2dc21aad/ language-es

Comisión de las Comunidades Europeas. (2015). Libro verde sobre los servicios financieros al por menor. Mejores productos, más posibilidades de elección y mayores oportunidades para consumidores y empresas. Consultado en https:// eur-lex.europa.eu/legal-content/ES/LSU/?uri=CELEX\%3A52015DC0630

Crane, A., McWillians, A., Matten, D., Moon, J. y Siegel, D. S. (2008). The Corporate Social Responsibility Agenda. En The Oxford Handbook of Corporate Social Responsibility. Oxford: Oxford University Press. Dor: https://doi.org/10.1093/ oxfordhb/9780199211593.003.0001

Cueto, C. (2014). La responsabilidad social corporativa del sector público. Múnich: GRIN Verlag. Consultado en https://www.grin.com/document/278423

Cueto, C. y De La Cuesta, M. (2017). La administración pública de la responsabilidad social corporativa. Coedición Madrid: UNED. Consutlado en http://portal.uned. es/Publicaciones/htdocs/pdf.jsp?articulo=0180266CO01A01

Cueva Estrada, M., Cueva Estrada, J. y Olivero Arias, E. (2015). Identificación de los stakeholders del banco nacional de fomento. Enciclopedia virtual: eumed.net. Consutlado en https://www.eumed.net/libros-gratis/2016/1506/ stakeholders.htm\#: :text=Cualquier\%20grupo\%20o\%20individuo\%20 que,(Freeman\%2C\%201984).

Deloitte. (2012). Análisis sobre la situación actual de las empresas españolas no cotizadas en relación con el Buen Gobierno. Consutlado en https://www2. deloitte.com/es/es/pages/governance-risk-and-compliance/articles/estudiodeloitte-esade-gobierno-corporativo.html

Drucker, P. F. (1984). The New Meaning of Corporate Social Responsibility. California Management Review, 26, 53-63.

Duque Orozco, Y. y Cardona Acevedo, M. (2013). Responsabilidad Social Empresarial: Teorías, índices, estándares y certificaciones. Cuadernos de administración, 29(50), 196-206. 
Elósegui Itxaso, M. (2010). El Libro verde de la Comisión Europea y la teoría de los stakeholders desde una perspectiva de género. Cuadernos Electrónicos de Filosofía del Derecho, 21, 1-38.

Ernst \& Young Global Limited. (2018). Novedades sobre información no financiera $y$ diversidad. Consutlado en https://go.ey.com/3k3Rv5h

Fernández Fernández, J. L. (2016). Un paradigma de la empresa para el siglo XxI. Cátedra de Ética Económica y Empresarial (Universidad Pontificia Comillas). Cuadernos de la Catedra, (10).

Fernández Fernández, J. y Bajo Sanjuán, A. (2012). La Teoría del Stakeholder o de los Grupos de Interés, pieza clave de RSE, del éxito empresarial y de la sostenibilidad. aDResearch: Revista Internacional de Investigación en Comunicación, 6(6), 130-143.

Forcadell, F. J. y Aracil, E. (2017). European banks' reputation for corporate social responsibility. Corporate Social Responsibility and Environmental Management, 24(1), 1-14.

Forética. (2018). Informe Forética 2018 sobre la evolución de la rse y sostenibilidad: la recompensa del optimista. Consutlado en https://www.foretica.org/informe_foretica_2018_resumen_ejecutivo.pdf

Franch, K. y Guerra, R. M. (2016). Las normas Iso 9000: una mirada desde la gestión del conocimiento, la información, innovación y el apredizaje organizacional. Cofin Habana, 10(2), 29-54.

Frederick, W. C. (1960). The Growing Concern Over Business Responsibility. California Management Review, 2, 54-61.

Freeman, R. (1984). Strategic management: A stakeholder approach. Pitman.

Freeman, R. E. y William, M. E. (1990). Corporate governance: A stakeholder interpretation. Journal of Behavioral Economics, 19(4), 337-359.

Friedman, M. (1962). Capitalism and freedom. Chicago: The University of Chicago Press.

Fundación Adecco. (2012). III Informe directivos y RC. https://fundacionadecco. org/memoria/2012.pdf

Fundación Corresponsables. (2019). Informe de sostenibilidad. Consultado en http:// publicaciones.corresponsables.com/publication/32e5d60d/mobile/ 
Fundación Ecología y Desarrollo. (2005). Anuario sobre la responsabilidad social en España. Zaragoza. Consultado en https://ecodes.org/documentos/archivo/ documentacion_sobre_rsc_ecodes_sep06.pdf

García-Santos, J. J. y Madero-Gómez, S. M. (2016). La evolución del concepto de responsabilidad social corporativa: revisión literaria. Conciencia Tecnológica, 51, 38-46. Consultado en https://dialnet.unirioja.es/servlet/ articulo? codigo $=6412980$

Garrigues Walker, A. y Trullenque Sanjuan, F. E. (2008). Responsabilidad social corporativa, ¿papel mojado o necesidad estratégica? Harvard Deusto business review, 164, 19-36.

Global Reporting Initiative. (2002). Global Reporting Initiative. Guia para la elaboración de memorias de sostenibilidad. Consultado en https://www.observatoriodeltrabajo.org/ftp/GRI.pdf

Global Reporting Initiative. (2016). GRI 101: Fundamentos 2016. Consultado en https://www.globalreporting.org/standards/media/1439/spanish-gri101-foundation-2016.pdf

Haller, A. y Van Staden, C. (2014). The value added statement - an appropriate instrument for integrated reporting. Accounting, Auditing and Accountability Journal, 27(7), 1190-1216.

Heras Saizarbitoria, I. y Arana Landín, G. (2013). La responsabilidad social corporativa y la norma sA 8000: un análisis de su adopción en las organizaciones cooperativas. Revista Vasca de Economía Social, 9, 31-52.

Huang, C. J. y Chao, M. H. (2001). Managing WWW in Public Administration: Uses and Misutes. Government Information Quarterly, 18(4), 357-373.

Kotler, P. y Lee, N. (2005). Corporate social responsibility: doing good for your company and your cause. Nueva Jersey: John Wiley \& Sons, Inc.

KPMG. (2011). KPMG International survey of corporate responsibility. KPMG International.

KPMG. (2017). The road ahead: KPMG survey of corporate responsibility reporting 2017. Consutlado en https://assets.kpmg/content/dam/kpmg/xx/pdf/2017/10/ kpmg-survey-of-corporate-responsibility-reporting-2017.pdf

Kreng, B. y Huang, M.-Y. (2011). Corporate social responsibility: Consumer behavior, corporate strategy, and public policy. Social behavior and personality: an internacional journal, 39(4), 529-541. 
Letza, S., Sun, X., y Kirkbride, J. (2004). Shareholding versus stakeholding: a critical review. Corporate Governance, 3(12), 242-262.

McWilliams, A. y Siegel, D. (2001). Corporate social responsibility: a theory of the firm perspective. Academy of Management Review, 26(1), 117-127.

Melé, D. (2007). Responsabilidad social de la empresa: una revisión crítica a las principales teorías. Ekonomiaz, 65, 50 -67.

Montero, M. (2012). La responsabilidad social y la norma Iso 26000. Revista de Formación Gerencial, 11(1), 102-119.

Morrós, J. y Vidal, I. (2005). Responsabilidad Social Corporativa. Madrid: Fundación Confemetal.

Moral, A. y Puentes-Poyatos, R. (2010). La responsabilidad social corporativa y su paralelismo con las sociedades cooperativas. Revista de Estudios Cooperativos, 103.

Mozas Mora, A. y Puentes Poyatos, R. (2010). La responsabilidad social corporativa y su paralelismo con las sociedades cooperativas. Revesco: Revista de estudios cooperativos, 103, 75-100.

Navarrete, J. y Gallizo, J. L. (2019). Reporte Integrado como herramienta de transparencia empresarial. Revista Academia y Negocios, 4(2), 71-82.

Navarro, A., Ortiz, D. y López, A. (2008). Identifying barriers to the application of standardized performance indicators in local government. Public Management Review, 10(2), 241-262.

Navarro, A., Alcaraz, F. J. y Ortiz, D. (2010). La divulgación de informaicón sobre Responsabilidad Corporativa en Administraciones Públicas: Un estudio empírico en gobiernos. Revista de Contabilidad, 13(2), 285-314.

Organización Internacional de Normalización. (2010). Guía de responsabilidad social: Iso 26000:2010(es). Consultado en https://www.iso.org/obp/ ui\#iso:std:iso:26000:ed-1:v1:es.

Otxoa-Errarte, R. y Landa-Oñati, J. (2012). Pyme y cooperativas ante el reto de la RSE: la nueva ISO 26000. Oñati Socio-Legal Series, 2(2), 57-79.

Pacto Mundial. (1999). Pacto Mundial de las Naciones Unidas: UN Global Compact. https://www.pactomundial.org/global-compact/

Palacios, C. y Coppa, C. (2015). Informe de sostenibilidad.Criterios y procedimientos en el mapeo de stakeholders. Un estudio de caso. Cuadernos de Contabilidad, 40(16), 233-259. 
Perdomo, J. y Escobar, A. (2011). La investigación en RSE: una revisión desde el management. Cuadernos de Administración, 24(43), 193-219.

Pinillos, A. A., y Fernández Fernández, J. L. (2011). De la RSC a la sostenibilidad corporativa: una evolución necesaria para la creación de valor. Harvard Deusto Business Review, 207, 1-14.

Pfeffer, J. (1993). Barriers to the advance of organizational science: Paradigm development as a dependent variable. Academy of Management Review, 18(4), 599-620.

Pina, V., Torres, L. y Yetano, A. (2009). Accrual Accounting in Eu local governments: one method, several approaches. European Accounting Review, 18(4), 765-807.

Pincheiro, A. P., Chiappetta, C. J. y Pereira, N. C. (2018). Corporate sustainability and value creation: the case of the "Dow Jones Sustainability Index". Gestão $y$ Producão, 25(3), 531-544.

Porter, M. y Kramer, M. (2011). Creating Shared Value. Harvard Business Review, 89(1-2), 62-77.

Puterman, P. (2010). Guía mundial de responsabilidad social. International CSR.

PWC. (2019). XXIII Encuesta Mundial de CEO. Consultado en https://www.pwc.es/ es/encuesta-mundial-de-ceos-2020.html

Responsables Consulting (2008). Libro verde sobre la responsabilidad social corporativa en el sector financiero: Una aproximación a la sostenibilidad desde las entidades financieras. Madrid: Confederación Española de Cajas de Ahorros.

Rivera-Arrubla, Y. A., Zorio-Grima, A. y García-Benau, M. A. (2016). El concepto de informe integrado como innovación en reporting corporativo. Journal of Innovation \& Knowledge, 1(3), 144-155.

Rodríguez, M. P., Caba, M. C. y López, A. M. (2007). E-Government and public financial reporting: the case of Spanish regional governments. American Review of Public Administration, 37(2), 142-177.

Rodríguez, L. A., Cuevas, V. y Aguirre, V. N. (2019). Responsabilidad Social Empresarial a las Responsabilidades en Derechos Humanos. Revista Política, Globalidad y Ciudadanía, 5(10), 63-79.

Rodríguez, J. M. (2003). El gobierno de la empresa: un enfoque alternativo. Madrid: Ediciones Akal.

Rodríguez, L. C. y Ríos-Osorio, L. A. (2016). Evaluación de sostenibilidad con metodología GRI. Dimensión Empresarial, 14(2), 73-89. 
Sethi, S. P. (1975). Dimensions of Corporate Social Responsibility. California Management Review, 17(3), 58-64.

Soyka, P. (2013). The International Integrated Reporting Council (IIRC). Integrated reporting framework: toward better sustainability reporting and (way) beyond. Environmental Quality Management, 23(2), 1-14.

Tarí, J., Molina-Azorín, J. y Heras, I. (2012). Benefits of the Iso 9001 and Iso 14001 standards: A literature review. Journal of Industrial Engineering and Management, 5(2), 297-322.

The International Framework. (2014). Marco Internacional $<I R>$. Consultado en https://integratedreporting.org/wp-content/uploads/2015/03/13-12-08-THEINTERNATIONAL-IR-FRAMEWORK-SPANISH-1.pdf

Weiss-Belalcázar, A. (2003). Responsabilidad social de las empresas en una sociedad de "afectados" (stakeholder society). Revista de Ciencias Administrativas y Sociales, 1(22), 43-54.

Wood, D. J. (1991). Corporate social performance revisited. Academy of Management Review, 16(4), 691-718.

Zorio-Grima, A., García-Benau, M. y Sierra-García, L. (2015). Aseguramiento del informe de sostenibilidad en España y Latinoamérica. Innovar: Revista de Ciencias Administrativas y Sociales, 25(extra 1), 85-102. 